\title{
Performance Analysis of CP-Based and CAZAC Training Sequence-Based Synchronization in OFDM System
}

\author{
R. Gaguk Pratama Yudha1, I Gede Puja Astawa², Amang Sudarsono³ \\ Politeknik Elektronika Negeri Surabaya \\ Jl. Raya ITS Politeknik Elektronika Negeri Surabaya \\ E-mail: gagukpratamayudha@yahoo.com ${ }^{1},\left\{\right.$ puja $^{2}$, amang $\left.^{3}\right\} @$ pens.ac.id
}

\begin{abstract}
Orthogonal Frequency Division Multiplexing (OFDM) is a popular wireless data transmission scheme. However, its synchronization is still being a major problem when it is applied in real hardware. Cyclic Prefix (CP) based synchronization is one of the solutions in this problem, but $\mathrm{CP}$ has high crest factor. In the other hand, CAZAC sequence is another solution with lower crest factor but the higher complexity and also CAZAC has potential in security and channel estimation implementation. The performance between CP and CAZAC sequence based synchronization in OFDM system is analyzed in this paper. The real hardware, Universal Software Rado Peripheral (USRP), is used to prove the analysis. The CAZAC sequence has $10 \%$ performance increased in frequency offset than CP based synchronization.
\end{abstract}

Keywords: OFDM, Synchronization, CAZAC, Training Symbol, Wireless Communication

\section{INTRODUCTION}

Orthogonal Frequency Division Multiplexing (OFDM) is a popular digital multicarrier modulation technique in wireless data transmission technology. It divides all of the data information into many of orthogonal subchannel. This OFDM can provide a very high data transmission rate compared to another modulation technique in a wireless transmission system. OFDM also has many advantages, such as high-frequency efficiency and robustness against Inter Symbol Interference (ISI) [11]. OFDM is also reliable in combating against the main property of wireless channels, such as fading. Nowadays, OFDM has been adopted in several modern communication technologies, such as Long Term Evolution (LTE), Digital Audio Broadcasting (DAB), Digital Video Broadcasting (DVB), and also Wireless Local Area Network (WLAN) IEEE 802.11 standard.

In addition to having a lot of advantages, OFDM also has several disadvantages. One of the disadvantages in OFDM system is its sensitivity against frequency and time mismatch [12]. A timing error is usually 
happened because the OFDM system failed to catch the transmitted signal in right time. This is usually affected by ISI when guard interval is too short and caused misalignment between one useful data with another useful data. This timing error will cause the demodulation system in OFDM cannot demodulate the data. A frequency error is usually caused by Doppler Shift effect and sometimes can also cause by unstabilities of the hardware local oscillator. This effect can cause the FFT system of OFDM cannot turn back the signal to frequency domain, and the data cannot be received perfectly in the receiver side.

The synchronization in OFDM systems have divided into data aided and non-data aided configuration [8]. The data aided configuration uses pilot sequence or training symbol to estimate the frequency and time mismatch of OFDM transmission. This method has high accuracy but losses the bandwidth and reduces the data transmission speed. The non-aided data uses a cyclic prefix, which is a last one-quarter of data, and position it in front of the data. This method doesn't decrease the bandwidth and doesn't reduces the data speed but the range of estimation is too small. This method is not suitable for data acquisition. In this paper, we describe and analyze two OFDM synchronization method, data aided and non-data aided.

\section{RELATED WORKS}

Many methods have been proposed by researcher all over the world to solve the OFDM synchronization problem. Scmidl and Cox [1] proposed the synchronization method using autocorrelation between two identical parts of training symbol to estimate the timing and frequency offset. This method successfully overcomes the synchronization problem, but this method causes long variance in timing estimation and reduces the data transmission rate of OFDM system. This method also uses a training symbol which caused the reduction of the space of data in one transmission OFDM packet. To improve the performance of scmidl technique, Minn [2] proposed the algorithm which exhibit shape and lower complexity compared to schmidl. Although this Minn algorithm can estimate the time and frequency offset better than Schmidl, the estimator is larger than guard interval in ISI channel. Ren [3] proposed a Constant Amplitude Zero Autocorrelation (CAZAC) based synchronization technique. Its performance is very good because it uses the CAZAC sequence which has the zero result of autocorrelation between two identical training symbols. But this method has a degradation of performance when it is applied in the Rayleigh Fading Channel Model. Meng [4] has proposed a new model of CAZAC based synchronization. Meng used the asymmetric model of training sequence which inrcease the robustness in Reyleigh Fading Channel. Silva[5], Silva had reviewed the configuration in short and long preambles of weighted preambles, based on CAZAC sequences weighted by a Pseudo Noise (PN) sequences. He designed short and long preambles with a CAZAC sequence weighted by a Golay Rudin Shapiro sequence and respective timing metrics. He simulated it in MATLAB, and get the Mean Square Error (MSE) of the 
estimation, the Bit Error Rate (BER) of transmitted data over Rayleigh fading channel with Additive White Gaussian Noise (AWGN), coarse timing and frequency offset.

\section{ORIGINALITY}

In this paper, we first analyze the performance comparison of two popular synchronization scheme. The first is CP based, and the second is training symbol based. Compared to Ren [3] and Meng [4] CAZAC, our CAZAC training symbol design is symmetric and can be changed over time. We use CAZAC sequence from silva(5), then implemented his modified CAZAC sequence in real hardware. This CAZAC sequence can provide the synchronization of OFDM system also can become a training symbol in channel estimation and a unique parameter for security purpose. As the conclusion, our CAZAC method can provide not only synchronization but also channel estimation and security system in OFDM transmission system. In the last, we implement this system in USRP as a real experimental testbed to investigate the performance in the real wireless channel.

\section{SYSTEM DESIGN}

We explain the system used in this paper in four sub-sections. The subsection 1 contains the description of OFDM system used in this paper. We use conventional OFDM system based on WLAN IEEE 802.11a OFDM standard. The subsection 2 contains the explanation of CP and Training Symbol based synchronization used. The subsection 3 contains the testing scenario of implemented system in real USRP hardware.

\subsection{OFDM System}

The OFDM system start from generating the information data. After the generation process, the data then is transformed to bits of data. This bits of data then is modulated using m-QAM modulation using bit mapper method. The result of this modulation is a serialized of symbol in frequency domain which is written as equation (1).

$S_{N}(n)=\left(S_{1}(n), S_{2}(n), S_{3}(n), \ldots\right]$

Where, $S(n)$ is data symbol, and $\mathrm{N}$ is the number of subcarrier. These serialized data symbol then are parallelized and inserted with a pilot symbol. The pilot symbol model used in this paper is block type. The ratio between pilot and the data is $1: 11$. The symbol that have been inserted with pilot symbol then are transformed to time domain using Inverse Fast Fourier Transform Method (IFFT). The number of IFFT is 64 where this is the same as the number of subcarrier. The result of this method is OFDM symbol in time domain as written in equation (2). 


$$
S_{m}(t)=\operatorname{Hin} T\left\{S_{n+p}(n)\right], t=1,2,3, \ldots, M
$$

The next step is serialized once again and insert cyclic prefix. The cyclic prefix is for conventional synchronization. For the training based synchronization, the training symbol is inserted as written in equation (3). The full explanation of synchronization process is explained in subsection 2 . The final output of OFDM transmitter is written in equation (4).

$$
S_{C,}(t)=\left\{\begin{array}{cc}
S(N+t), t=-N g,-N g+1, \ldots \\
S(t), \quad t=0,1, \ldots, N-1
\end{array}\right.
$$

$$
S_{c y}(t)=-S_{c p}(t), S_{:}(t), S_{2}(t), S_{3}(t), \ldots, S_{i n}(t), \quad t=1,2,3,4, \ldots, N-1
$$

Where, $S(N+t)$ is data plus the last $1 / 4$ of data. The final OFDM symbol is $S_{c x}(t)$ in time domain.

In the receiver, after the OFDM symbol has been received, the Cyclic Prefix or Training symbol of the data is removed. After that, the data is turned back to the frequency domain by using FFT method. For Channel Estimation method, we use Least Square (LS) channel estimation because of its low complexity and easy implementation in real hardware. The LS channel estimation equation is written in equation (5).

$h_{i s}=\frac{Y_{p}(k)}{X_{p}(k)}$

Where, Yp is the received pilot symbol, and Xp is determined pilot symbol. $h_{i s}$ is the channel state estimation parameter. To detect and reformat the data we use Zero Forcing (ZF) detector. This ZF detector work by multiplying the data with estimated channel parameter $h_{i s}$ as written in equation (6).

$t(k)=\left(h_{L S}{ }^{H} h_{L S}\right)^{-2} h_{L S}{ }^{n} \times \bar{y}$

Where, $t(k)$ is the data after detecting method, and $\bar{y}$ is the data received in the receiver side. After this method, the last method is demodulate the detected data, and information and be extracted from them. The all of these OFDM block systems is shown in figure 1 . 


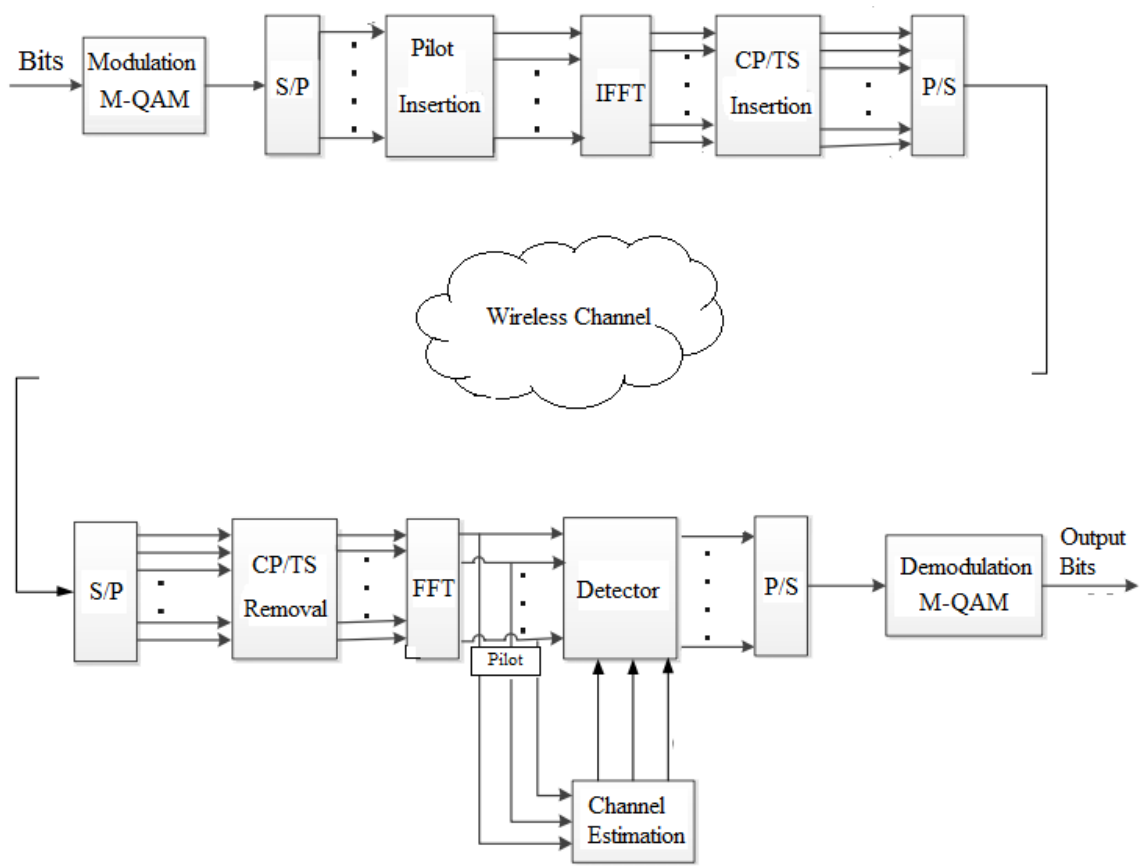

Figure 1. Full OFDM Block Diagram

\subsection{Synchronization in OFDM System}

In this subsection, we describe 2 different synchronization methods. The first is CP based synchronization method, and the second is CAZAC training based synchronization method.

\subsubsection{CP Based Synchronization Method}

In this method, the CP is inserted in front of the data. The CP is taken from the last $1 / 4$ of the one packet data. Figure 2 shows the CP insertion in OFDM packet.

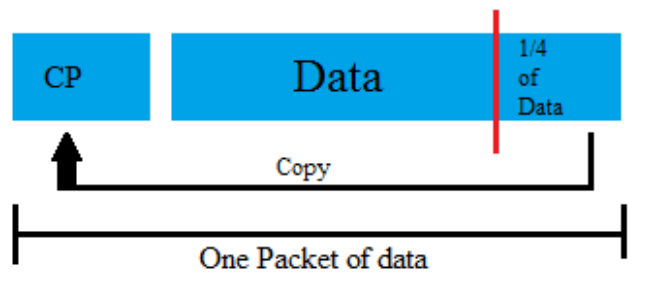

Figure 2. CP Insertion in OFDM packet Data

To estimate the time offset, Schmidl and Cox [1], use autocorellation between the last $1 / 4$ of data and the CP itself. If the autocorrelation process is resulting 'matched', the CP then is extracted and the data is processed further. If the autocorrelation's result is not 'matched', this synchronization method will 
keep searching until it gets the matched autocorrelation. Figure 3 shows the graphical explanation of this process.
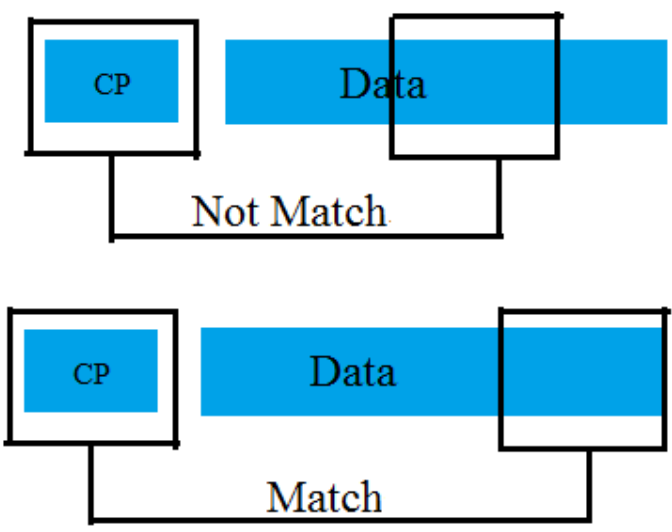

Figure 3. Time Synchronization using Sliding Window

Assume $\mathrm{S}$ is the CP of the OFDM system, according to [5], it can be represented as in (7).

$S(k)=e^{\frac{i, i+k^{2}}{i}}$

Where $\mathrm{j}$ is imaginary number and $\mathrm{M}$ is relative prime of $\mathrm{N}$. $\mathrm{N}$ itself is a number of subcarrier. When there is a frequency offset in the system, the received signal will is represented in (8).

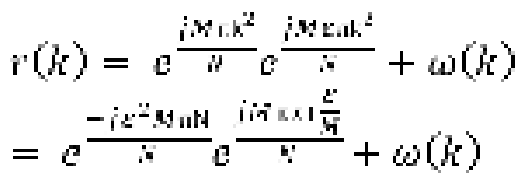

The relationship between timing deviation and frequency offset is used to find the fine timing and the CFO. We assume that $\varepsilon$ is an integer value of CFO in range of $\left|\varepsilon-\frac{1}{2}\right|<|e|<\left|\varepsilon+\frac{1}{2}\right|$, the equation (8) can be changed to equation (9).

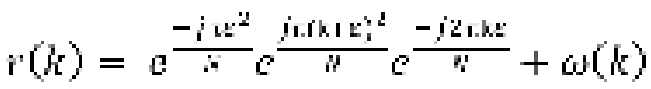

As shown in (9), $e^{\frac{-i_{x} i^{2}}{n}}$ is constant, it will only change the amplitude of the signal, but the CFO will cause a corresponding deviation in timing. 


\subsubsection{CAZAC Training Symbol Based Synchronization Method}

In the second synchronization method, CAZAC sequence is used as Training Symbol (TS). Assume that $\mu(k)$ is a CAZAC sequence with length of $\mathrm{N}$, the property of it is represented in (10).

$$
\begin{aligned}
& |\mu(k)|=C_{1} \quad k=0,1,2,3, \ldots \\
& C(m)=\sum_{k=1}^{n-1} \mu(m+k) \mu(k)=0, \quad m=1,2,3, \ldots, N-1
\end{aligned}
$$

The CAZAC sequence property does not change in IFFT or FFT process, so that in OFDM symbol CAZAC sequence still a CAZAC sequence. Figure shows the CAZAC sequence.

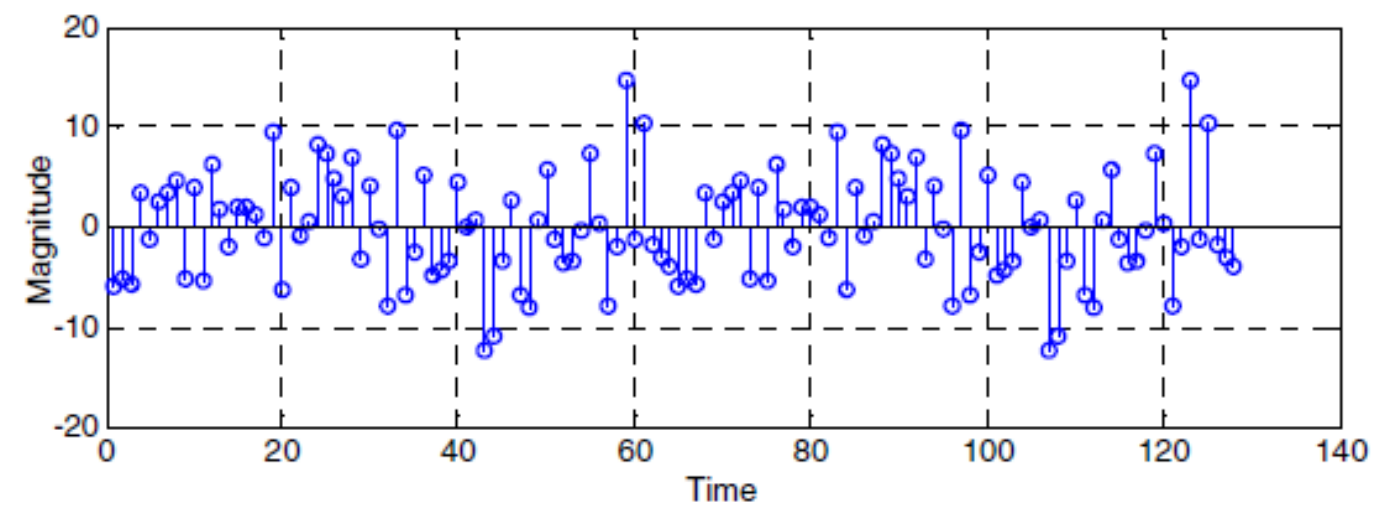

Figure 4. CAZAC Sequence in Matlab Simulation

To calculate the Time Offset and Carrier Frequency Offset (CFO), this method use autocorrelations between received and known CAZAC sequence which is represented in (11).

$$
\begin{aligned}
& M(d)=\sum_{k=1}^{n} r_{y}(d+k) s^{\prime}(k)
\end{aligned}
$$

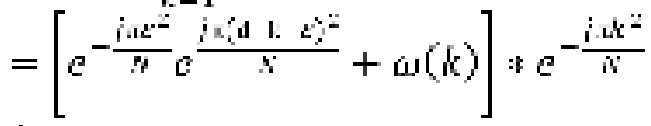

$$
\begin{aligned}
& \bar{d}=\arg \max (k(d))
\end{aligned}
$$

Where $M(d)$ is the cross-correlation of the received TS and the known TS in the receiver. $\bar{d}$ is the estimation of timing start point. According to [7], CAZAC 
will get the max value at $\vec{d}=d_{c u r}-\tau$, and $d_{c u r}$ is the correct start point of TS.

The next step is to calculate the autocorrelation of the received signal rs(k) and the known TS as in (12).

$$
\begin{aligned}
& G(k)=r_{S}(k)+S^{-}(k) \\
& =c^{-\frac{j, k^{2}}{i n} e^{\frac{j 21 . k E}{\|}}+\omega(k)} \\
& \omega(k)=\omega(k): c^{-\frac{j 1 . k^{2}}{N}}
\end{aligned}
$$

Where $\omega(k)$ is the mean of fractional CFO and $\varepsilon$ is CFO itself. The whole receiver block diagram with synchronization is shown in figure 5 .

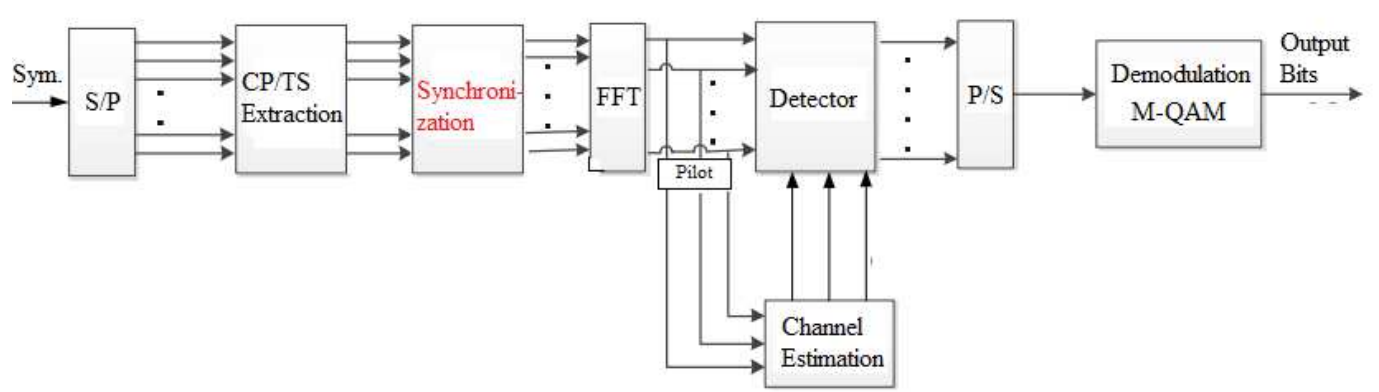

Figure 5. The receiver Block Diagram of OFDM System

\section{Performance Evaluation}

For the investigation of this two methods, we use USRP. USRP is set inside the laboratory which has a size of $8 \times 8$ meters. We use 2 distance parameters in this paper, one is 2-meter distance and the second is 5-meter distance. For 2 meter distance, the signal to noise ratio (SNR) is $80 \mathrm{~dB}$. For 5 meter distance, the signal to noise ratio is $55 \mathrm{~dB}$. First, we apply the CP-based OFDM and try to challenge the CP based synchronization by unsynchronize the time and the frequency. We unsynchronize the time in OFDM system by adding another frame in front of the usual frame beside of the real unsynchronization because of real channel. We also unsynchronize the carrier frequency by changing the subcarrier frequency to test the synchronization method. Figure 6 shows the testing scenario of the USRP. 


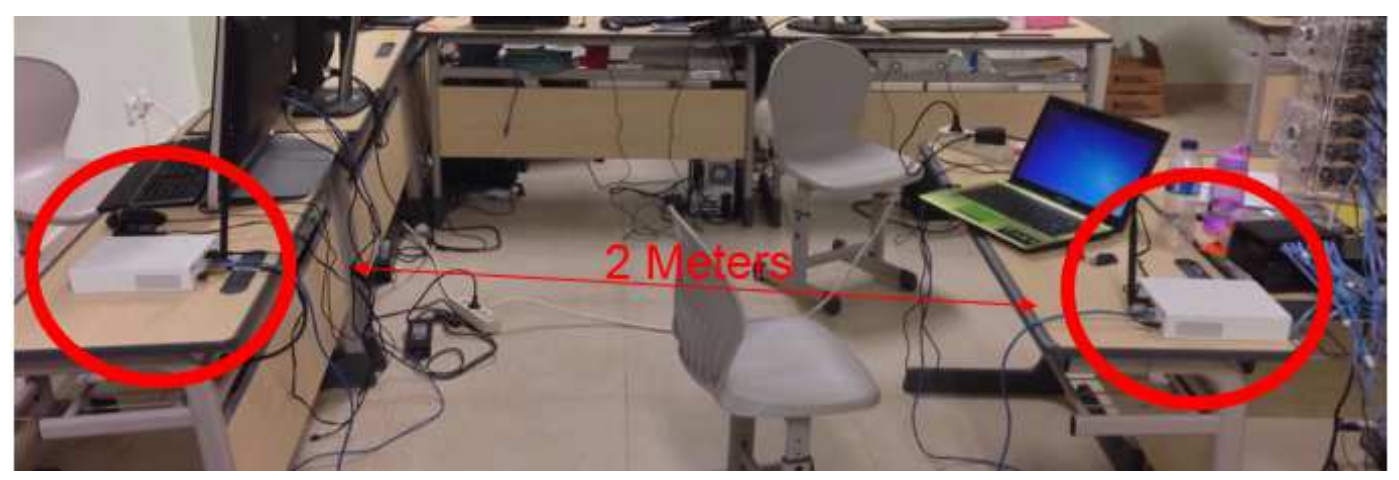

Figure 6. The Real Testing Scenario using USRP

We try to mimic IEEE 802.11a standard in this scenario [10]. We use 52 number of subcarrier. When we use CP Based synchronization, there are 12 more data that will be used, it means the total data transmitted will be 64 . For modulation scheme, we use 4-QAM, 16-QAM, and 64 QAM. We mapped a bit to symbol based on constellation diagram. For the center frequency, we use band $900 \mathrm{MHz}$. All the testing scenario parameter is described in table 1.

Table 1. Testing Parameters

\begin{tabular}{|c|c|}
\hline Parameters & Value \\
\hline Number of Subcarrier & 52 \\
\hline Number of FFT & 64 \\
\hline Cyclic Prefix (CP) ratio & $1 / 4$ \\
\hline Modulation & $4 \sim 64 \mathrm{QAM}$ \\
\hline Center Frequency & $915 \mathrm{MHz}$ \\
\hline Number of Transmitter & 1 Antenna \\
\hline Number of Receiver & 1 Antenna \\
\hline Distance Variation (m) & $2 \mathrm{~m}$, and 5m \\
\hline Equalizer & ZF \\
\hline Synchronization & CP and CAZAC TS Based \\
\hline Channel & Indoor Channel \\
\hline Signal to Noise Ratio (SNR) & $26 \& 50 \mathrm{~dB}$ \\
\hline Dimension Laboratory & $8 \mathrm{~m}$ x 8m \\
\hline
\end{tabular}

The next step is to compare the constellation diagram in receiver side. We compare the constellation to see the performance of CP and CAZAC TS synchronization. We use 4-QAM, 16-QAM, and 64-QAM as the OFDM system modulation. The comparation of the constellation from this scenario is shown in table 2. 
Table 2. The Constellation Comparison between CP and CAZAC based Synchronization

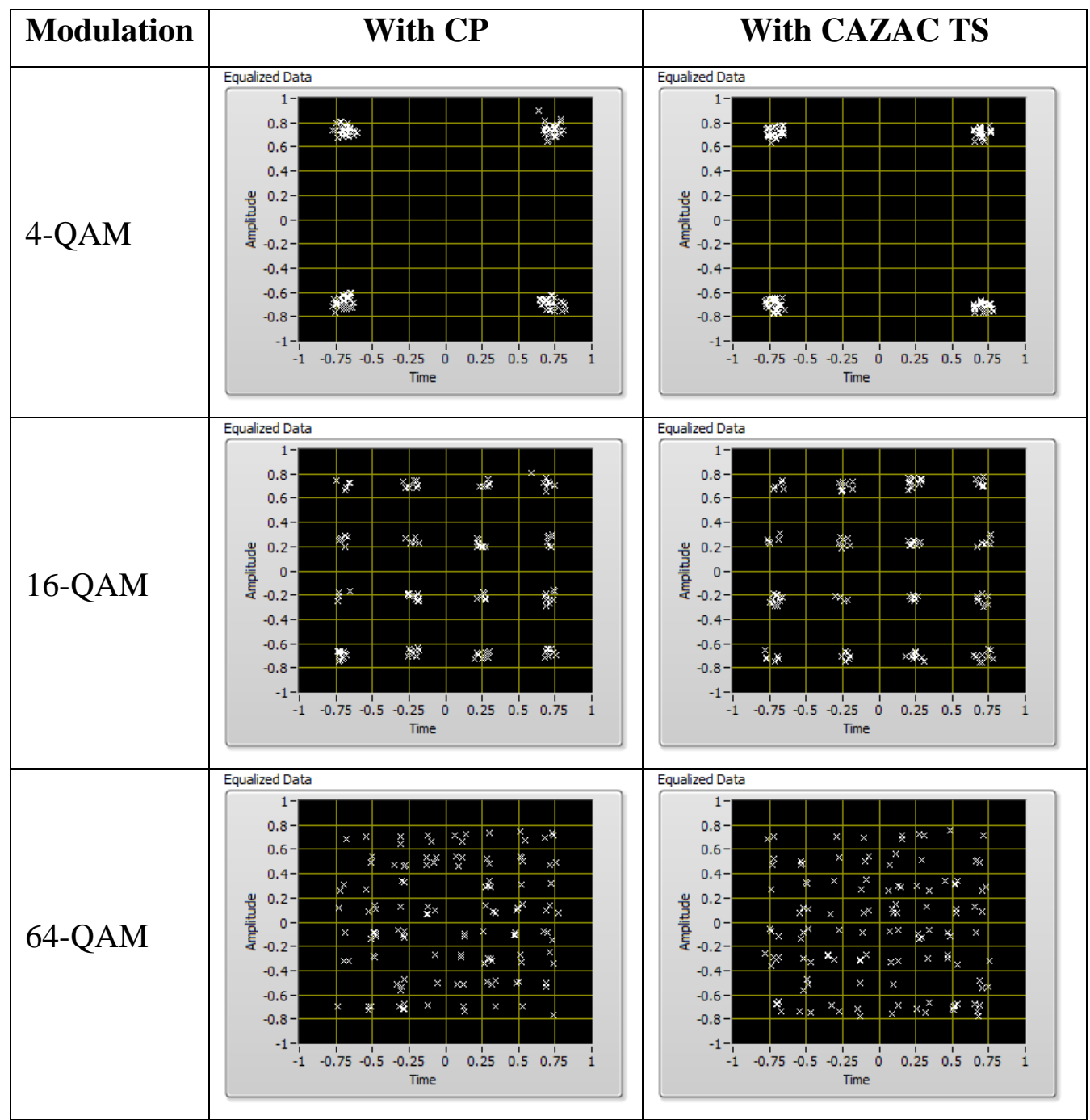

From the constellation diagram, we can see that the performance of this two methods is almost same. For further investigation, we add the sample offset for testing the synchronization performance of these two methods. We assume that the data is offset but there is not any data lost. We analyze the comparison by using Bit Error Graph (BER) graph. Figure 7 shows the BER vs Sample Offset comparison between CP and CAZAC synchronization. 


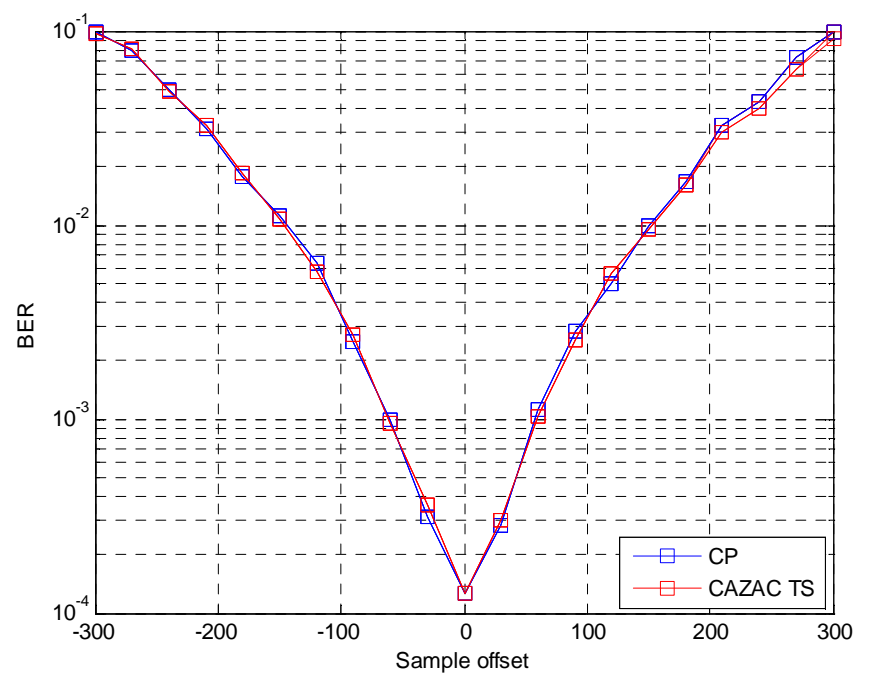

Figure 7. BER vs Sample Offset of CP and Cazac Synchronization

Table 3. Data BER Sample offset CP and CAZAC

\begin{tabular}{|l|l|l|}
\hline Sample & \multicolumn{1}{|c|}{ CP } & \multicolumn{1}{c|}{ Cazac TS } \\
\hline-300 & $1 \times 10^{-1}$ & $1 \times 10^{-1}$ \\
\hline-200 & $7,8 \times 10^{-1}$ & $7,9 \times 10^{-1}$ \\
\hline-100 & $8,1 \times 10^{-2}$ & $8,4 \times 10^{-2}$ \\
\hline 100 & $8,1 \times 10^{-2}$ & $8,4 \times 10^{-2}$ \\
\hline 200 & $7,8 \times 10^{-1}$ & $7,9 \times 10^{-1}$ \\
\hline 300 & $1 \times 10^{-1}$ & $1 \times 10^{-1}$ \\
\hline
\end{tabular}

The performance of $\mathrm{CP}$ and CAZAC TS synchronization in time synchronization is almost same. There are only small differences between them. By using CAZAC TS, performance is increased by $10 \%$ in sample offset of 300. The next analysis is by comparing the constellation of CP and CAZAC in the condition of sample time offset of 100 samples. Figure 8 shows the constellation diagram of CP and CAZAC Based Synchronization with 100 offset samples.
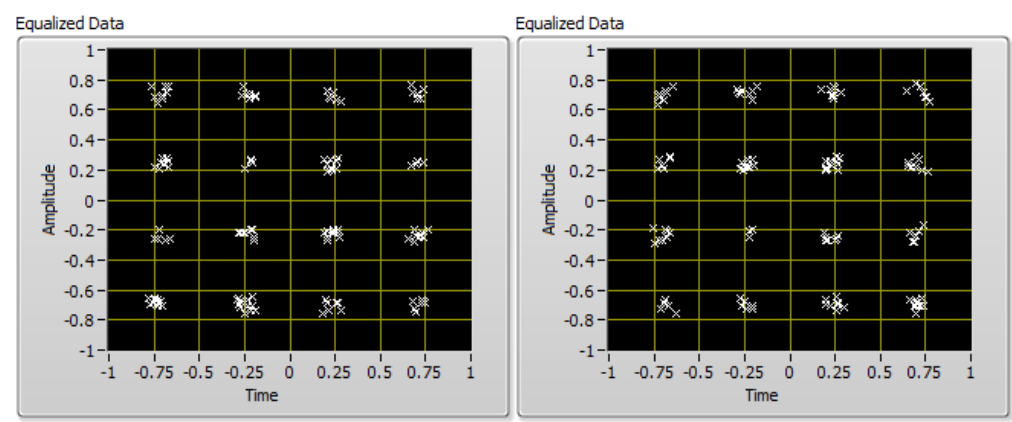

Figure 8. Constellation of CP and CAZAC synchronization by adding sample time offset of 100 respectively 
The last investigation is to investigate the Carrier Frequency Offset between two methods. We compare CP and CAZAC synchronization by using BER graph. We test the performance in zero frequency offset until $10 \mathrm{kHz}$ frequency offset. Figure 9 shows the BER vs Frequency offset comparison between two methods.

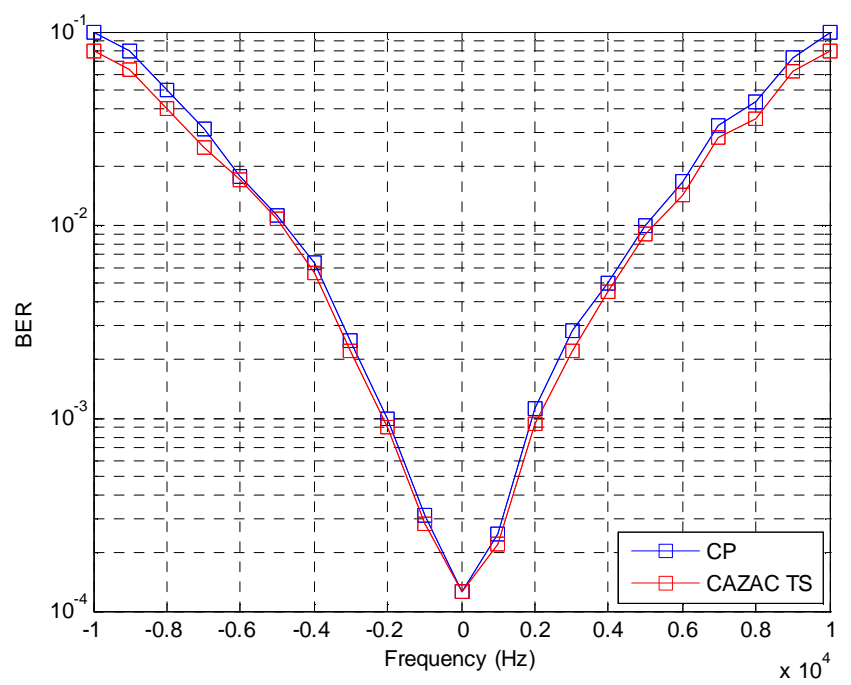

Figure 9. BER vs Sample Offset of CP and CAZAC Synchronization

Table 4. Data BER frequency $\mathrm{CP}$ and CAZAC

\begin{tabular}{|l|l|l|}
\hline \multicolumn{1}{|c|}{ Freq } & \multicolumn{1}{c|}{ CP } & \multicolumn{1}{c|}{ Cazac TS } \\
\hline$-1 \times 10^{4}$ & $1 \times 10^{-1}$ & $3 \times 10^{-1}$ \\
\hline$-0,8 \times 10^{4}$ & $5 \times 10^{-1}$ & $6 \times 10^{-1}$ \\
\hline$-0,6 \times 10^{4}$ & $8 \times 10^{-1}$ & $8,4 \times 10^{-1}$ \\
\hline$-0,2 \times 10^{4}$ & $1 \times 10^{-3}$ & $3 \times 10^{-3}$ \\
\hline $0,2 \times 10^{4}$ & $1 \times 10^{-3}$ & $3 \times 10^{-3}$ \\
\hline $0,6 \times 10^{4}$ & $9,2 \times 10^{-1}$ & $9,5 \times 10^{-1}$ \\
\hline $1 \times 10^{4}$ & $1 \times 10^{-1}$ & $3 \times 10^{-1}$ \\
\hline
\end{tabular}

The BER graph in figure 9 shows that the performance of CAZAC has outperformed $\mathrm{CP}$ in frequency offset of $10 \mathrm{kHz}$. Comparing with $\mathrm{CP}$, the performance of CAZAC is increased around $15 \%$. We also investigate the performance based on the constellation diagram. Figure 10 shows the constellation diagram of CP and CAZAC with 16-QAM modulation. 


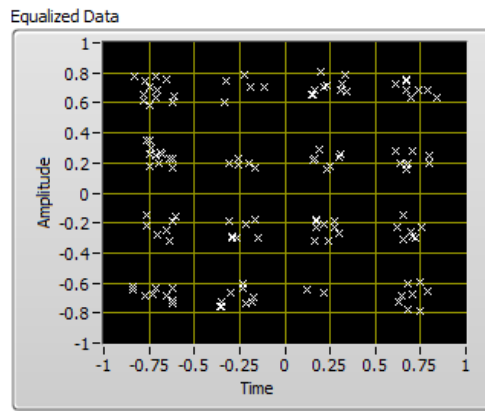

(a)

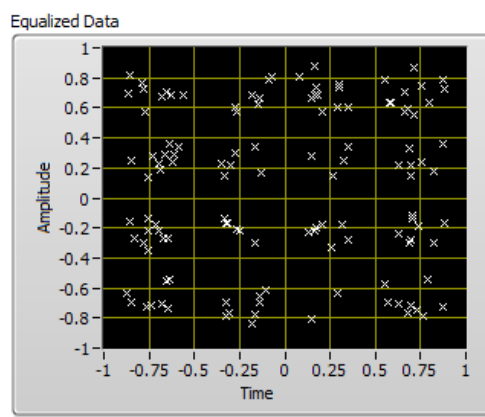

(c)

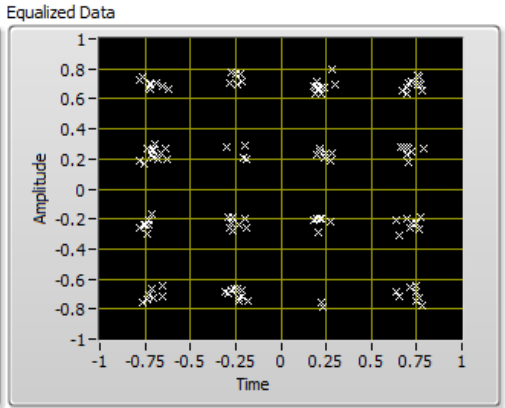

(b)

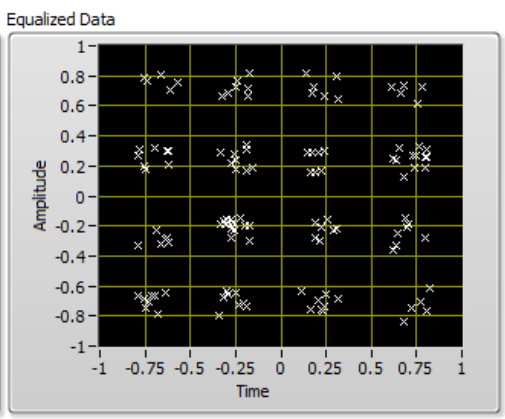

(d)

Figure 10. Constellation of $\mathrm{CP}$ and CAZAC synchronization by adding frequency offset of $200 \mathrm{~Hz}(\mathrm{a}-\mathrm{b})$ and $10 \mathrm{kHz}$ (c-d) respectively

The constellation of CP based is more scattered than the constellation of CAZAC. In the frequency offset of $200 \mathrm{~Hz}$, the spread of constellation between two is almost the same. But, in $10 \mathrm{kHz}$ frequency offset, the constellation of CAZAC sequence is better than $\mathrm{CP}$ based synchronization. In distance between 2 meters and 5 meters BER performance shows with CAZAC TS Synchronization is better than CP. For ensuring that CAZAC method is better than $\mathrm{CP}$ we add investigation measurement on 2 meters and 5 meters to provide a barrier from cardboard between $\mathrm{Tx}$ and $\mathrm{Rx}$. The experimental result that CAZAC method is still better than CP. Figure $\mathbf{1 2}$ showed that the blue line is CAZAC method and the red line is CP method. For ensuring that CAZAC method is better than CP we add investigation measurement on 2 meters and 5 meters to provide a barrier from cardboard between Tx and Rx. The experimental result that CAZAC method is still better than CP. 


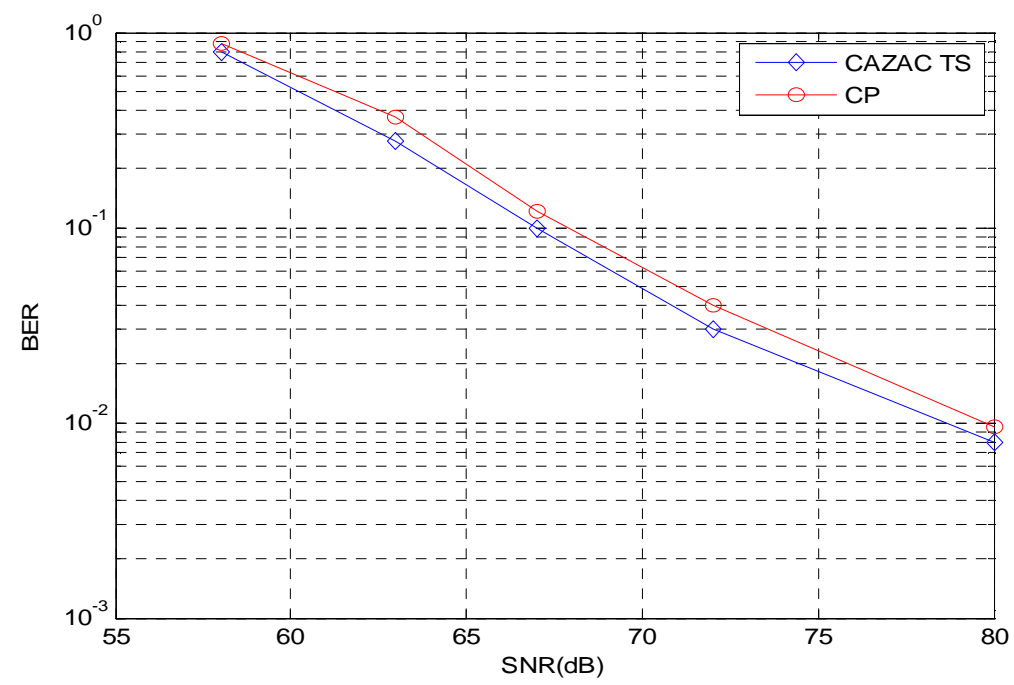

Figure 11. BER Performance between CP and CAZAC Based Synchronization

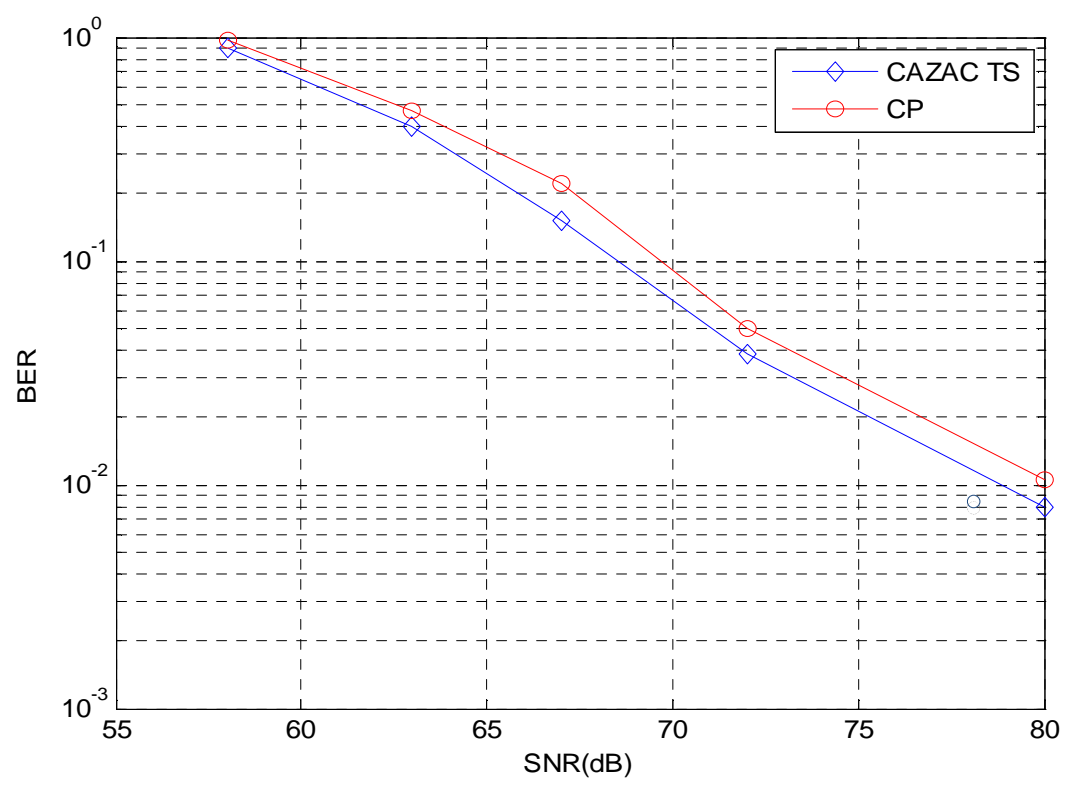

Figure 12. BER performance with obstacle CP and CAZAC Based Synchronization

\section{Conclusion}

In this paper, the performance of $\mathrm{CP}$ Based synchronization and CAZAC TS based synchronization is investigated. These two methods have similar performance in high SNR, but in low SNR, the CAZAC TS based synchronization is better. These two methods can combat frequency offset and time offset error, and CAZAC based synchronization outperform CP. By 
using CAZAC TS, performance is increased by $10 \%$ in sample offset of 300 . CAZAC also outperform CP in frequency offset error of $10 \mathrm{kHz}$. Comparing with $\mathrm{CP}$, the performance of CAZAC is increased around $15 \%$. In low SNR, the CAZAC has better performance that $\mathrm{CP}$ about $2 \mathrm{~dB}$.

\section{References}

[1] T. M. Scmidl, D. C. Cox, Robust Frequency and Timing Synchronization for OFDM, IEEE Transactions of Communications, Vol. 45, No. 12, pp. 1613-1621, 1997.

[2] H. Minn, V. K. Bhargava, K. B. Letaief, A Robust Timing and Frequency Synchronization for OFDM Systems, IEEE Transactions on Wireless Communications, Vol. 2, No. 4, pp. 822-839, 2003.

[3] G. Ren, Y. Chang, H. Zhang, and H. Zhang, Synchronization Method Based On a New Constant Envelop Preamble for OFDM systems, IEEE Transactions of Broadcasting, Vol. 51, No. 1, pp. 139-143, 2005.

[4] J. Meng, G. Kang, A Novel OFDM Synchronization Algorithm Based on CAZAC Sequence, International Conference on Computer Application and System Modeling, Taiyuan, pp. 1634-1637, 2010.

[5] E. M. Silva C., F. J. Harris, G. J. Dolecek, Synchronizations Algorithm based on Weighted CAZAC Preambles for OFDM Systems, International Symposium on Communications and Information Technologies, Surat Thani, pp. 192-197, 2013.

[6] M. Ruan, M. C. Reed, Z. Shi, Training Symbol Based Coarse Timing Synchronization in OFDM Systems, IEEE Transactions of Wireless Communication, Vol. 8, No. 5, pp. 2558-2569, 2009.

[7] O. Omomukuyo, D. Chang, J. Zhu, O. Dobre, R. Venkatesan, T. Ngatched. C. Rumbolt, Joint Timing and Frequency Synchronization based on Weighted CAZAC Sequences for Reduced Guard Interval CO-OFDM Systems, Optics Express, Vol.23, No. 5, pp. 1-12, 2015.

[8] L. Deneire, B. Gyselinckx, M. Engels, Training Sequence versus Cyclic Prefix - A New Look on Single Carrier Communication, IEEE Communications Letter, Vol. 5, No. 7, pp. 292-294, 2001.

[9] L. Lei, C. Song, T. Zhang, Performance Evaluation for OFDM Link Based on LabVIEW and USRP, Instrumentation and Measurement, Computer, Communication and Control (IMCCC), Harbin, pp. 897-901, 2014.

[10] The Institute of Electrical and Electronic Engineers, Inc., IEEE Standard of Information Technology, IEEE Press (New York), 802.11-2012, 2012.

[11] L. Litwin, M. Pugel, The Principal of OFDM, RF Signal Processing [online], Microsoft Research, pp. 30-48, 2001. 
[12] C. R. N. Athaudage, BER Sensitivity of OFDM System to Time Synchronization Error, The $8^{\text {th }}$ International Conference on Communication System, pp. 42-46, 2002.

[13] J.Brillhart,P.Morton, "A Case Study in Mathematical Research: The Golay-Rudin-Shapiro Sequence," American Mathematical Monthly, Vol103, Part 10, pp 854-869, 1996. 